\title{
Multiwavelength evidence for a 15-year periodic activity in the symbiotic nova V1016 Cygni ${ }^{\star}$
}

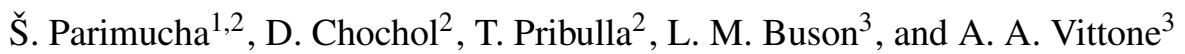 \\ ${ }^{1}$ Faculty of Natural Sciences, Department of the Theoretical Physics and Geophysics, University of P. J. Šafárik, \\ 04001 Košice, Slovakia \\ 2 Astronomical Institute of the Slovak Academy of Sciences, 05960 Tatranská Lomnica, Slovakia \\ 3 INAF Osservatorio Astronomico di Capodimonte, via Moiariello 16, 80131 Napoli, Italy
}

Received 26 March 2002 / Accepted 4 June 2002

\begin{abstract}
The $\sim 15.1$ years period found in the long-term $U B V$ photoelectric and photographic photometry of the symbiotic nova V1016 Cyg is detected also in the $(J-K)$ colour index and in the UV continuum and emission line fluxes from IUE and HUT spectra. It could be interpreted either as the effect of recurrent enhanced mass loss episodes from the Mira type variable companion to a hot component along its ultra-wide orbit (proposed from recent HST observations) or the true orbital period of the inner, unresolved binary of a triple system. The 410-day delay of the maximum of UV emission lines fluxes with respect to the maximum of continuum was found. The pulsation period of the Mira type variable was improved to $474 \pm 6$ days.
\end{abstract}

Key words. stars: binaries: symbiotic - stars: individual: V1016 Cyg - stars: activity

\section{Introduction}

V1016 Cyg (MH $\alpha$ 328-116) is a member of a small subgroup of symbiotic stars, called symbiotic novae, also including V1329 Cyg and HM Sge, whose outbursts lead to a nebular spectrum (Mürset \& Nussbaumer 1994). Symbiotic novae are wide interacting binaries, where matter from a late-type giant is transferred onto the surface of the more compact companion. The nova-like optical outburst ( $\Delta m \sim 5-7 \mathrm{mag}$ ), lasting decades, is caused by a thermonuclear runaway on the surface of a wind-accreting white dwarf after the critical amount of material has been accumulated (cf. Mikolajewska \& Kenyon 1992). V1016 Cyg underwent such nova-like outburst in 1964 (McCuskey 1965). The object is classified as a D-type symbiotic, the cool component being a Mira type variable embedded in a dust envelope whose pulsation period turned out to be $\sim 478$ (Munari 1988). The onset of a dust formation episode in 1983 is reported by Taranova \& Yudin (1986).

The orbital period of V1016 Cyg is not yet established. Taranova \& Yudin (1983) made use of the increase of Balmer emission lines in combination with the appearance and disappearance of FeII lines to derive an orbital period of $\approx 20$ years.

Send offprint requests to: Š. Parimucha, e-mail: parimuch@ta3.sk

* Partly based on observations obtained with the International Ultraviolet Explorer (IUE) satellite retrieved from the IUE Newly Extracted Spectra (INES) Archive and the Hopkins Ultraviolet Telescope (HUT) retrieved from the Multimission Archive at STScI (MAST).
Afterwards, Nussbaumer \& Schmid (1988), though unable of recording two consecutive maxima, proposed an orbital period of 9.5 years on the basis of the apparent periodicity seen in the flux of OI and MgII UV emission lines by means of the IUE satellite. At the same time Munari (1988), by resorting to IR observations taken over two decades, proposed instead a 6-year orbital period by modeling the sequence of dust obscuration episodes, likely related to the passage of the Mira type variable at the inferior conjunction in the system.

Much longer periods have been proposed by Wallerstein (1988) and Schild \& Schmid (1996). In the former paper the author, assuming that the sharp FeII emission lines are formed in the chromosphere of the cool star so as to reflect its orbital motion, concludes that their observed radial velocities between 1978 and 1985 limit any high inclination orbit to a period greater than 25 years or to a large eccentricity. The latter analysis, based on spectropolarimetric data taken from 1991 to 1994, indicates that the orbital period is about $80 \pm 25$ years, though later observations obtained in 1997 put this result into question (Schmid 1998). Finally, Brocksopp et al. (2002), adopting a projected separation of the two stellar components as large as $84 \mathrm{AU}$ on the basis of their HST/WFPC2 images, are forced to propose an astonishingly long orbital period of $\sim 544$ years.

In the context of this still-to-be-settled issue, one has also to interpret the evidence for the 15-year periodic activity (not necessarily representing the sought orbital period) recently presented by Parimucha et al. (2000) (hereafter PAC) who 


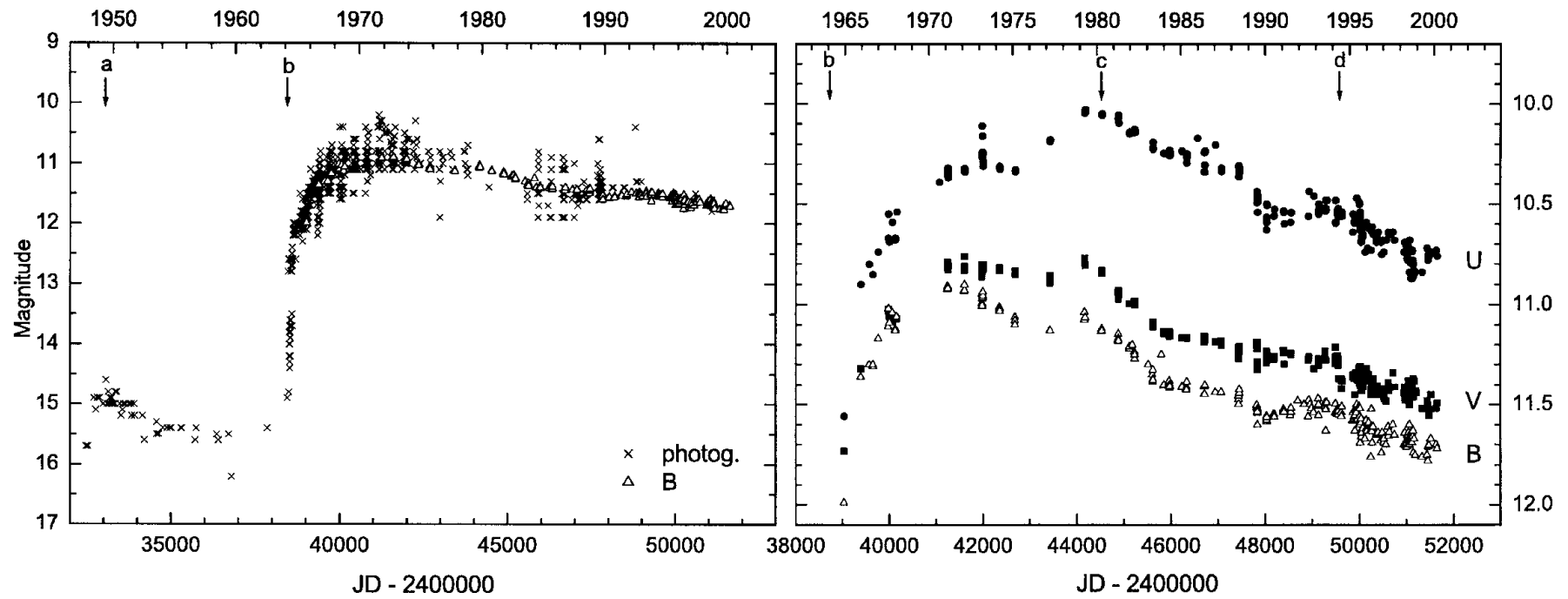

Fig. 1. Photographic (left) and $U B V$ (right) light curves of V1016 Cyg. Arrows $a, b, c$ and $d$ mark the epochs of subsequent activity episodes of the system (see text).

gathered and analysed long-term photographic, photoelectric and visual photometry of the object. The aim of the present paper is indeed to give further (i.e., multiwavelength) support to the existence of such a periodicity making use of both IR photometry and UV spectroscopy, as well as to investigate its own origin.

\section{Long-term photometry}

\subsection{UBV photoelectric and photographic data}

The historical light curve of V1016 Cyg based on the photographic and $U B V$ data given in PAC is presented in Fig. 1. The light curve suggests four stages of activity marked by arrows in the figure: the pre-outburst flare $a$ in 1949, the main nova-like outburst $b$ in 1964 and two post-outburst, decreasing-amplitude flares $c$ and $d$ in 1980 and 1994, respectively. Evidently the activity episodes affecting the system repeat themselves (though at quite a different intensity level) with an interval of $\approx 15$ years. The ephemeris for the activity maxima calculated in PAC is as follows:

$$
\begin{gathered}
\mathrm{JD}_{\max }^{\text {phot }}=2427590+5510 \times E . \\
\pm 250 \quad \pm 90
\end{gathered}
$$

It is worth noticing that both maxima recorded in 1980 and 1994 followed appreciable brightness decreases which, in turn, could be interpreted as signatures of an enhanced mass transfer from the cool to the hot component. A similar effect has been detected in the light curve of the very slow classical nova V723 Cas (Chochol \& Pribulla 1998).

\subsection{JHK data}

Infrared photometry of V1016 Cyg was published by Harvey (1974), Kenyon \& Gallagher (1983), Lorenzetti et al. (1985), Taranova \& Yudin (1986), Munari (1988), Ananth \& Leahy (1993), Kamath \& Ashok (1999) and Taranova \& Schenavrin (2000). We make use here of this whole dataset plus a few unpublished $J H K$ observations obtained in 1993 (kindly provided by F. Strafella) to newly estimate the period of pulsation of the Mira type variable. The Fourier period analysis (Fig. 2) applied to the $J H K$ data leads to the values $476 \pm 10,472 \pm 11$ and $474 \pm 10$ days for $J, H$ and $K$ photometry, respectively. The adopted period of the Mira type variable pulsations (whose corresponding phase diagrams are also presented in Fig. 2) is thus $P=474 \pm 6$ days.

The long-term behaviour of the $(J-K)$ color index of V1016 Cyg using all available infrared data is presented in Fig. 3. As pointed out by Whitelock (1987), the $(J-K)$ color index in symbiotic Miras is little affected by the Mira type variable pulsation, being conversely very sensitive to the amount of circumstellar dust around the cool component. As a consequence, the abrupt change $(\sim 1 \mathrm{mag})$ of the IR color recorded in 1983 by Taranova \& Yudin (1986) comes likely from a short (though strong) dust formation episode. Although orbitally-related dust obscuration episodes are indeed expected in these systems, we interpret this unique, major episode which occurred three years after the observed maxima both in the $U$ passband and space-borne UV (see below) as dust formation in the ejecta of the symbiotic nova (cf. Bode 1995).

According to this view, one has to be cautious when interpreting possible periodicities emerging from the analysis of the long-term behaviour of the $(J-K)$ color index (Fig. 3) which shows an additional maximum in 1988 and a clear minimum in 1992, together with a more uncertain maximum (in 1973) and a further possible minimum (in 1977). Indeed, the phenomena lying beneath the two possible periods (namely, 2070 and 5630 days; see Fig. 4) one can identify by means of the Fourier analysis of its residuals (after removing a parabolic trend) is likely quite different. While the longer period, besides being close to the $U B V$ photometric estimate, could well reflect the real orbital motion of the system, as suggested by the wavelike variation exhibited by the $(J-K)$ residuals phased with the ephemeris (1) (see Fig. 4), the shorter period of 5.6 years, close to the 6-year period interpreted by Munari (1988) as the orbital 

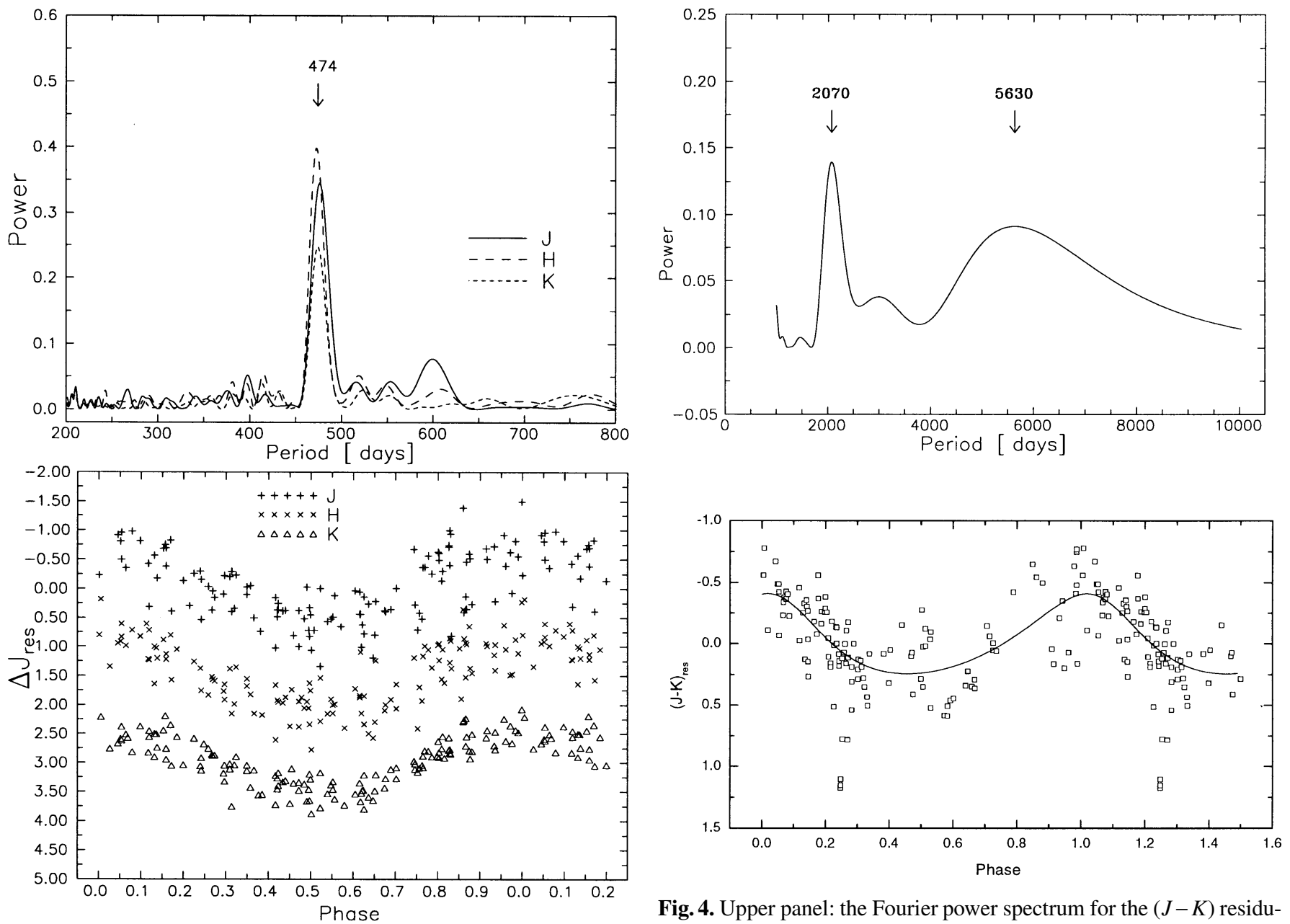

Fig. 4. Upper panel: the Fourier power spectrum for the $(J-K)$ residuals in the interval 1000 to 10000 days. Lower panel: the phase diagram of the above data corresponding to the ephemeris (1). A trigonometric polynomial has been adopted for the fit.

Fig. 2. Upper panel: the Fourier power spectra of the pulsation period of the Mira type variable in the $J H K$ data. Lower panel: the resulting light curves phased with the ephemeris $\mathrm{JD}_{\max }=2447442+474 \times E$. The $\Delta H_{\text {res }}$ and $\Delta K_{\text {res }}$ are shifted by 1.5 and $3.0 \mathrm{mag}$, respectively.

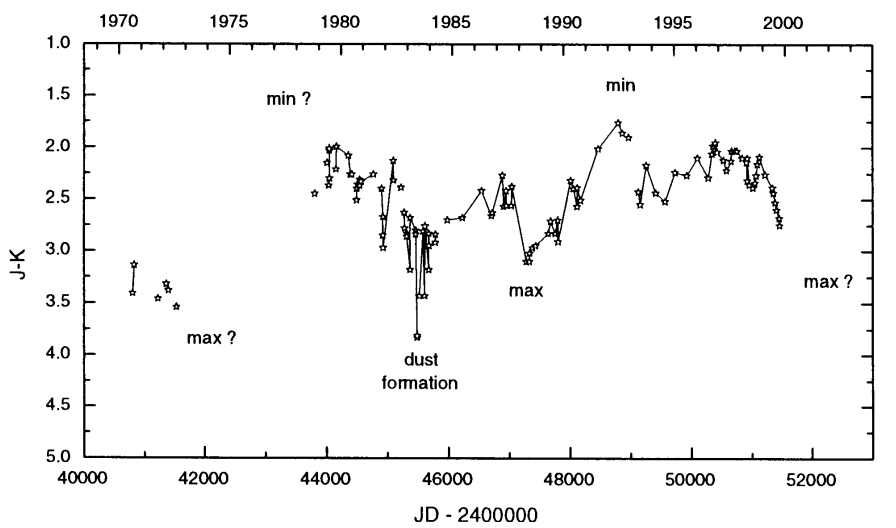

Fig. 3. $(J-K)$ color index of V1016 Cyg.

period of the binary system, is likely due to the superposition of several effects such as dust formation induced by the activity of the hot component and possible mass tranfer events from the cool to the hot component.

\section{UV spectroscopy}

The UV dataset discussed here includes both low and high dispersion IUE (International Ultraviolet Explorer) spectra spanning the interval from Aug. 1978 to Aug. 1995 (for a complete list see Parimucha 2002), and a single HUT (Hopkins Ultraviolet Telescope) spectrum taken on March 6, 1995. Properly calibrated IUE NEWSIPS data have been retrieved from the IUE Newly Extracted Spectra (INES) Archive, while the calibrated HUT spectrum was extracted from the Multimission Archive at STScI (MAST).

Average continuum fluxes in emission-free, $20 \AA$ bins centered at $\lambda 1840 \AA$ and $\lambda 2530 \AA$ have been derived from low dispersion, large aperture IUE spectra (SWP and LWR/LWP cameras, respectively), plus the HUT spectrum (for the $\lambda 1840 \AA$ region alone). Both measured and dereddened fluxes are given in Table 1 (having adopted $E(B-V)=0.28$, according to Nussbaumer \& Schild 1981). Dereddened continuum fluxes are also shown in Fig. 5.

High dispersion, large aperture IUE/SWP spectra were used to derive fluxes of OIII] $(\lambda \lambda 1661,1666 \AA)$, NIII] $(\lambda 1750 \AA), \operatorname{SiIII}](\lambda 1892 \AA)$ and CIII] $(\lambda 1909 \AA)$ emission lines. A single measurement of the OIII] lines flux was obtained 
Table 1. Measured (I) and corrected (II) (with $E(B-V)=0.28$ ) UV continuum flux in $10^{-14} \mathrm{erg} \mathrm{cm} \mathrm{s}^{-1} \AA^{-1}$.

\begin{tabular}{|c|c|c|c|c|c|c|}
\hline JD & \multicolumn{3}{|c|}{$1840 \AA$} & \multicolumn{3}{|c|}{$2530 \AA$} \\
\hline $2400000+$ & SWP & I & II & LWR/P & I & II \\
\hline 43752 & 2427 & 15.8 & 122.1 & 2229 & 16.5 & 102.6 \\
\hline 43920 & 4271 & 15.4 & 119.1 & 3778 & 18.4 & 114.0 \\
\hline 44049 & 5611 & 16.9 & 130.6 & & & \\
\hline 44072 & 5832 & 17.6 & 136.1 & 5080 & 20.7 & 128.3 \\
\hline 44076 & & & & 5136 & 22.6 & 140.1 \\
\hline 44139 & & & & 5675 & 19.9 & 123.3 \\
\hline 44140 & 6617 & 16.3 & 125.9 & & & \\
\hline 44202 & & & & 6227 & 20.9 & 129.5 \\
\hline 44268 & 7803 & 13.9 & 107.4 & & & \\
\hline 44474 & & & & 8593 & 20.8 & 128.9 \\
\hline 44475 & 9878 & 16.0 & 123.7 & & & \\
\hline 44672 & 13431 & 15.0 & 115.9 & 10095 & 19.1 & 118.4 \\
\hline 44706 & & & & 10344 & 17.8 & 110.3 \\
\hline 44707 & 13704 & 13.8 & 106.7 & & & \\
\hline 44761 & 14193 & 12.4 & 95.8 & 10786 & 19.6 & 121.5 \\
\hline 45065 & & & & 12966 & 20.2 & 125.2 \\
\hline 45192 & & & & 13920 & 17.9 & 110.9 \\
\hline 45193 & 17658 & 11.9 & 91.9 & & & \\
\hline 45301 & 18669 & 14.2 & 109.7 & 14733 & 15.9 & 98.6 \\
\hline 45422 & 19566 & 12.4 & 95.8 & 15597 & 14.6 & 90.5 \\
\hline 45799 & & & & 3133 & 12.6 & 78.1 \\
\hline 45800 & 22701 & 10.0 & 77.3 & & & \\
\hline 45822 & & & & 3261 & 12.8 & 79.3 \\
\hline 46045 & 24656 & 11.7 & 90.4 & 4959 & 12.0 & 74.4 \\
\hline 46616 & & & & 8552 & 12.8 & 79.3 \\
\hline 46771 & & & & 9656 & 11.9 & 73.8 \\
\hline 46935 & 31006 & 11.1 & 85.8 & 10793 & 12.6 & 78.1 \\
\hline 47111 & 32296 & 12.6 & 97.4 & 12065 & 13.2 & 81.8 \\
\hline 47332 & 33783 & 11.4 & 88.1 & 13464 & 12.8 & 79.4 \\
\hline 47512 & 35047 & 11.8 & 91.2 & 14651 & 12.6 & 78.1 \\
\hline 47750 & & & & 16106 & 12.9 & 79.9 \\
\hline 47751 & 36825 & 12.0 & 92.7 & & & \\
\hline 47777 & 36953 & 9.7 & 74.9 & 16298 & 14.9 & 92.3 \\
\hline 47855 & & & & 16822 & 10.6 & 65.7 \\
\hline 48004 & & & & 17793 & 13.1 & 81.2 \\
\hline 48005 & 38658 & 10.2 & 78.8 & & & \\
\hline 48122 & 39486 & 12.0 & 92.8 & 18609 & 12.7 & 78.7 \\
\hline 48420 & & & & 20582 & 17.3 & 107.2 \\
\hline 48472 & 42161 & 10.9 & 84.3 & 20934 & 12.4 & 76.8 \\
\hline 48613 & & & & 22055 & 14.9 & 92.4 \\
\hline 48614 & 43445 & 13.2 & 102.1 & & & \\
\hline 48814 & & & & 23483 & 16.0 & 99.2 \\
\hline 48917 & 46028 & 13.9 & 107.4 & 24127 & 16.0 & 99.2 \\
\hline 49514 & & & & 28380 & 15.3 & 94.9 \\
\hline 49782 & $11301^{a}$ & 15.7 & 121.3 & & & \\
\hline 49955 & & & & 31358 & 14.2 & 88.1 \\
\hline 49956 & 55707 & 12.0 & 92.8 & & & \\
\hline
\end{tabular}

Note: ${ }^{a}$ HUT spectrum.

also from the HUT spectrum of 1995 . The emission line profiles show a broad component superposed to the dominant, narrow component (cf. Kindl et al. 1982). Fluxes given here are the result of fitting Gaussian profiles to the latter narrow

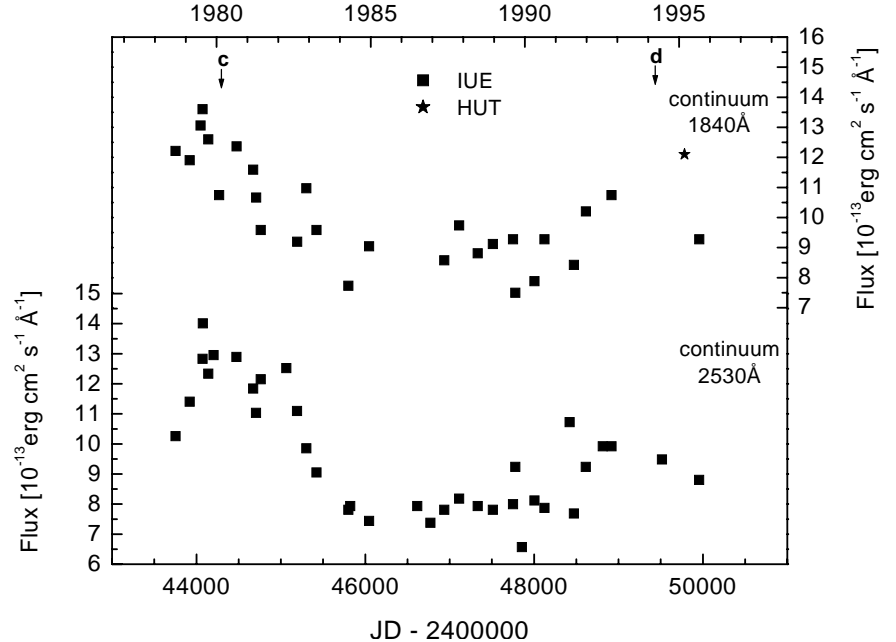

Fig. 5. Dereddened continuum fluxes at $1840 \AA$ and $2530 \AA$.

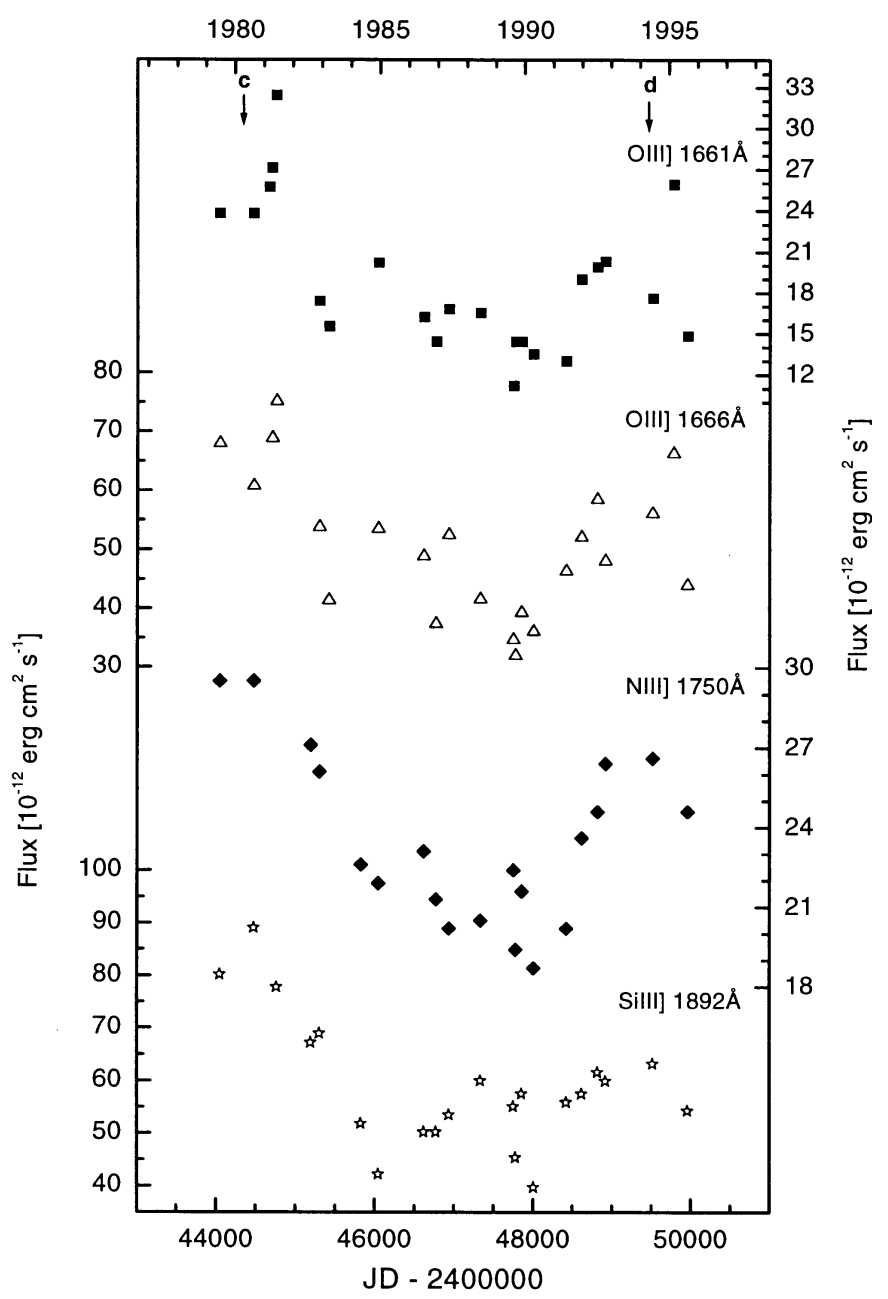

Fig. 6. Dereddened UV emission line fluxes.

component. Dereddened fluxes for OIII], NIII] and SiIII] lines are shown in Fig. 6 and listed (together with the original fluxes) in Table 2.

For the strong CIII] $\lambda 1909 \AA$ which falls by chance in the overlapping region between the IUE short-wavelength (SWP) and long-wavelength (LWR/LWP) camera spectral ranges, 
Table 2. Measured (I) and corrected (II) (with $E(B-V)=0.28$ ) UV emission line fluxes in $10^{-12} \mathrm{erg} \mathrm{cm}^{-2} \mathrm{~s}^{-1}$.

\begin{tabular}{|c|c|c|c|c|c|c|c|c|c|}
\hline \multirow{2}{*}{$\frac{\mathrm{JD}}{2400000+}$} & \multirow[t]{2}{*}{ SWP } & \multicolumn{2}{|c|}{ OIII]1661 Å } & \multicolumn{2}{|c|}{ OIII]1666 ^̊ } & \multicolumn{2}{|c|}{ NIII] $1750 \AA$} & \multicolumn{2}{|c|}{ SiIII]1892 Å } \\
\hline & & I & II & I & II & I & II & I & II \\
\hline 44049 & 5612 & 3.2 & 23.8 & 9.1 & 68.0 & 3.7 & 29.5 & 9.9 & 80.2 \\
\hline 44475 & 9879 & 3.2 & 23.8 & 8.1 & 60.8 & 3.7 & 29.5 & 11.0 & 89.1 \\
\hline 44672 & 13432 & 3.4 & 25.7 & & & & & & \\
\hline 44707 & 13705 & 3.6 & 27.1 & 9.2 & 68.9 & & & & \\
\hline 44761 & 14192 & 4.3 & 32.4 & 10.0 & 75.1 & & & 9.6 & 77.8 \\
\hline 45193 & 17657 & & & & & 3.4 & 27.1 & 8.3 & 67.2 \\
\hline 45301 & 18670 & 2.3 & 17.4 & 7.2 & 53.8 & 3.3 & 26.1 & 8.5 & 68.9 \\
\hline 45422 & 19568 & 2.1 & 15.5 & 5.5 & 41.4 & & & & \\
\hline 45823 & 22890 & & & & & 2.8 & 22.6 & 6.4 & 51.8 \\
\hline 46045 & 24658 & 2.7 & 20.2 & 7.2 & 53.5 & 2.7 & 21.9 & 5.2 & 42.2 \\
\hline 46617 & 28618 & 2.2 & 16.2 & 6.5 & 48.9 & 2.9 & 23.1 & 6.2 & 50.2 \\
\hline 46771 & 29823 & 1.9 & 14.4 & 5.0 & 37.4 & 2.7 & 21.3 & 6.2 & 50.2 \\
\hline 46935 & 31005 & 2.2 & 16.8 & 7.0 & 52.5 & 2.5 & 20.2 & 6.6 & 53.5 \\
\hline 47332 & 33785 & 2.2 & 16.5 & 5.6 & 41.6 & 2.6 & 20.5 & 7.4 & 60.0 \\
\hline 47751 & 36826 & 1.5 & 11.2 & 4.6 & 34.7 & 2.8 & 22.4 & 6.8 & 55.1 \\
\hline 47777 & 36954 & 1.9 & 14.4 & 4.3 & 32.0 & 2.4 & 19.4 & 5.6 & 45.4 \\
\hline 47855 & 37673 & 1.9 & 14.4 & 5.3 & 39.3 & 2.7 & 21.6 & 7.1 & 57.5 \\
\hline 48005 & 38659 & 1.8 & 13.5 & 4.8 & 36.1 & 2.3 & 18.7 & 4.9 & 39.7 \\
\hline 48420 & 41828 & 1.7 & 13.0 & 6.2 & 46.4 & 2.5 & 20.2 & 6.9 & 55.9 \\
\hline 48614 & 43446 & 2.5 & 19.0 & 7.0 & 52.1 & 2.9 & 23.6 & 7.1 & 57.5 \\
\hline 48815 & 45115 & 2.7 & 19.9 & 7.8 & 58.5 & 3.1 & 24.6 & 7.6 & 61.6 \\
\hline 48917 & 46029 & 2.7 & 20.3 & 6.4 & 48.1 & 3.3 & 26.4 & 7.4 & 59.9 \\
\hline 49514 & 51059 & 2.4 & 17.6 & 7.5 & 56.1 & 3.3 & 26.6 & 7.8 & 63.2 \\
\hline 49782 & $11301^{a}$ & 3.4 & 25.9 & 8.8 & 66.3 & & & & \\
\hline 49956 & 55705 & 2.0 & 14.8 & 5.9 & 44.0 & 3.1 & 24.6 & 6.7 & 54.3 \\
\hline
\end{tabular}

Note: ${ }^{a}$ HUT spectrum.

simultaneous flux estimates obtained from both cameras are shown in Fig. 7 and listed in Table 3. In order to make the best use of the whole dataset, Fig. 7 includes also - as a lower limit - the estimated flux of CIII] in case the line saturation was marginal (i.e. affecting no more than 2 high-resolution pixels).

It should be stressed that the availability of a large aperture spectrum taken on August 31, 1978 (namely, LWR 2228), allowing the measurement of the CIII] $\lambda 1909 \AA$ emission line flux, does assure that the flux rise exhibited by the UV continua from mid-1978 to mid-1979 (see Fig. 5) also affects the UV emission lines so as both UV continua and emission lines reflect the 1980 peak of activity of the system recorded in other wavebands (see above). Strictly speaking, this verification turns out to be not possible for the remaining set of UV emission lines, as high resolution SWP spectra assured earlier than June 24, 1979 were obtained through the non-photometric IUE small aperture.

One can easily recognize that the evolution of both UV continua and emission lines from 1978 to 1996 is characterized by two maxima matching the activity epochs $\mathrm{c}$, $\mathrm{d}$ shown by optical photometry. Moreover, interesting clues to the physical properties of the system could come from the observed delay of the onset of emission line activity, whose first maximum (at epoch JD $2444635 \pm 15$ ) appears shifted by 410 days from the corresponding epoch for both UV continua (JD $2444225 \pm 12$ ).

Finally, it should hold reader's interest the fact that the major dust formation episode discussed in the Sect. 2.2 reflects in

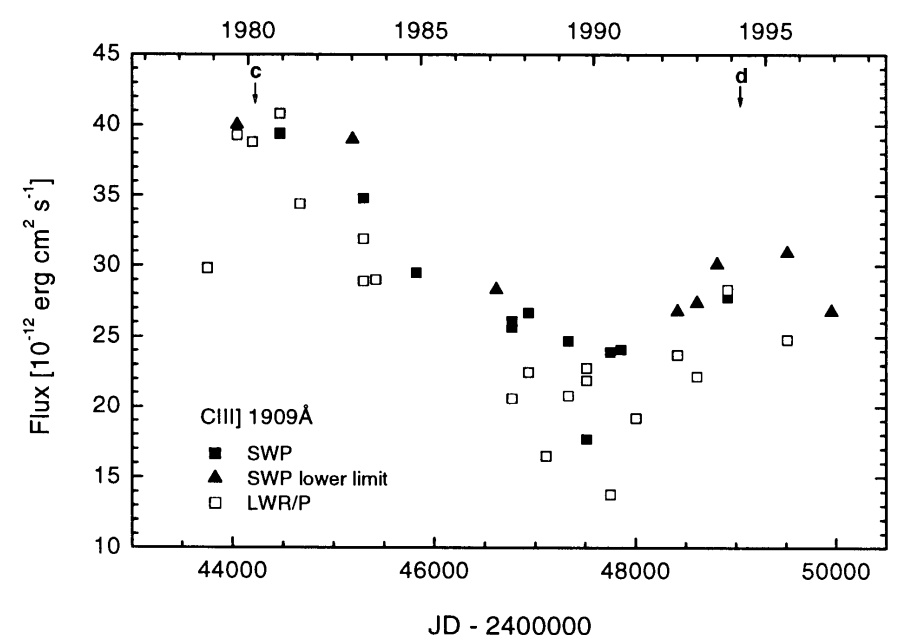

Fig. 7. Dereddened fluxes of CIII] $\lambda 1909 \AA$ emission line.

a recognizable flux drop of the majority of UV emission lines in 1983-84 (see Fig. 6).

\section{Discussion}

No doubt the symbiotic nova V1016 Cyg includes a pulsating Mira type variable and an accreting white dwarf that underwent a thermonuclear outburst in 1964, leading straight to a nebular spectrum (FitzGerald et al. 1966). According to the ionization 
Table 3. Measured (I) and corrected (II) (with $E(B-V)=0.28$ ) CIII]1909 ̊ emission line fluxes in $10^{-11} \mathrm{erg} \mathrm{cm}^{-2} \mathrm{~s}^{-1}$ from SWP and LWP/R spectra.

\begin{tabular}{ccccccc}
\hline \hline JD & SWP & I & II & LWR/P & I & II \\
$2400000+$ & & & & & & \\
\hline 43752 & & & & 2228 & 3.6 & 29.8 \\
44049 & $5612^{1}$ & 4.8 & 40.0 & 4869 & 4.7 & 39.3 \\
44203 & & & & 6228 & 4.7 & 38.8 \\
44475 & 9879 & 4.7 & 39.4 & 8594 & 4.9 & 40.8 \\
44672 & & & & 10096 & 4.1 & 34.4 \\
45193 & $17657^{1}$ & 4.7 & 39.0 & & & \\
45301 & 18670 & 4.2 & 34.8 & 14734 & 3.8 & 31.9 \\
45301 & & & & 14735 & 3.5 & 28.9 \\
45422 & & & & 15599 & 3.5 & 29.0 \\
45823 & 22890 & 3.6 & 29.5 & & & \\
46617 & $28618^{1}$ & 3.4 & 28.3 & & & \\
46771 & 29823 & 3.2 & 26.1 & 9646 & 2.4 & 19.5 \\
46771 & & & & 9647 & 2.5 & 20.6 \\
46771 & 29828 & 3.1 & 25.7 & & & \\
46935 & 31005 & 3.2 & 26.7 & 10794 & 2.7 & 22.5 \\
47111 & & & & 12066 & 2.0 & 16.5 \\
47332 & 33784 & 3.0 & 24.7 & 13463 & 2.5 & 20.8 \\
47512 & 35046 & 2.1 & 17.7 & 14652 & 2.7 & 22.8 \\
47512 & & & & 14653 & 2.6 & 21.9 \\
47751 & 36826 & 2.9 & 23.9 & 16108 & 1.7 & 13.8 \\
47855 & 37673 & 2.9 & 24.1 & & & \\
48005 & & & & 17795 & 2.3 & 19.2 \\
48420 & $41828^{1}$ & 3.2 & 26.8 & 20583 & 2.9 & 23.7 \\
48614 & $43446^{1}$ & 3.3 & 27.4 & 22056 & 2.7 & 22.2 \\
48815 & $45115^{1}$ & 3.6 & 30.1 & & & \\
48917 & 46029 & 3.4 & 27.8 & 24128 & 3.4 & 28.3 \\
49514 & $51059^{1}$ & 3.7 & 30.9 & 28379 & 3.0 & 24.8 \\
49956 & $55705^{1}$ & 3.2 & 26.8 & & & \\
\hline & & & & & &
\end{tabular}

Note: ${ }^{1}$ Lower limit.

model of symbiotic binaries, the hot luminous component ionizes the neutral wind of the giant giving rise to the nebula in the system. Such a mechanism is confirmed by the strict coincidence of the epochs of maxima in the $U$ passband (observed in 1980 and 1994) and those recorded in the space-UV continuum combined with the 410-day delay of the OIII], CIII], NIII] and SiIII] emission line maxima which, in turn, assures that the lines are collisionally excited in the surrounding nebula when the fast wind from the hot object interacts with the slow wind from the Mira type variable.

At present, the above well-understood phenomenology leaves still open two alternative scenarios for V1016 Cygni.

If this symbiotic nova is a wide-orbit binary with a 544-year period as proposed by Brocksopp et al. (2002), one is forced to interpret the periodic (15-yr) variations of the optical and UV continuum as induced by flares of the hot component triggered by the recurrent enhanced mass loss episodes from the Mira type variable companion. According to Fleischer et al. (1995), this mass loss is most pronounced every few periods of the Mira type variable pulsations. It can trigger individual flares in the accretion disk of the hot white dwarf. The existence of the disk is supported by the 3D simulation of the wind accretion by the compact star (Theuns \& Jorissen 1993).

Alternatively, V1016 Cygni could be interpreted as a triple system, whose inner, unresolved binary has an orbital period of 15 years. If this is the case, flares would be triggered by an enhanced mass transfer from a cool giant during its periastron passage on the 15-year orbit. In this respect, a hint of enhanced mass transfer, coming just before the weak flare in 1994, can be found in the long-term infrared photometry performed by Taranova \& Schenavrin (2000) showing a brightness increase in the $J$ and $H$ passbands in 1992. Moreover, the coincidence of the observed maximum of the $J-K$ index in 1988 (Fig. 3) and the wide minima of the UV continua (Fig. 5) would constrain to that epoch the inferior conjuction of a cool giant along its 15-year orbit.

Acknowledgements. This work was accomplished as a part of a Ph.D. Thesis of Š.P. and has been supported by VEGA Grant 2/1157 of the Slovak Academy of Sciences. The authors would like to thank to F. Strafella for providing his unpublished IR observations and A. Skopal for critical reading of the manuscript.

\section{References}

Ananth, A. G., \& Leahy, D. A. 1993, J. Astrophys. Astron., 14, 37 Bode, M. F. 1995, in Wolf-Rayet Stars: Binaries, Colliding winds, Evolution, ed. K. A. van der Hucht, \& P. M. Williams (Kluwer, Dordrecht), 363

Brocksopp, C., Bode, M. F., Eyres, S. P. S., et al. 2002, ApJ, in press Chochol, D., \& Pribulla, T. 1998, Contrib. Astron. Obs. Skalnaté Pleso, 28, 121

FitzGerald, M. P., Houk, N., McCuskey, S. W., \& Hoffleit, D. 1966, ApJ, 144, 1135

Fleischer, A. J., Gauger, A., \& Sedlmayr, E. 1995, A\&A, 297, 543

Harvey, P. M. 1974, ApJ, 188, 95

Kamath, U. S., \& Ashok, N. M. 1999, A\&AS, 135, 199

Kenyon, S. J., \& Gallagher, J. S. 1983, AJ, 88, 666

Kindl, C., Marxer, N., \& Nussbaumer, H. 1982, A\&A, 116, 265

Lorenzetti, D., Saraceno, P., \& Strafella, F. 1985, ApJ, 298, 350

McCuskey, S. 1965, IAU Circ., 1916

Mikolajewska, J., \& Kenyon, S. J. 1992, MNRAS, 256, 177

Munari, U. 1988, A\&A, 200, L13

Mürset, U., \& Nussbaumer, H. 1994, A\&A, 282, 586

Nussbaumer, H., \& Schild, H. 1981, A\&A, 101, 118

Nussbaumer, H., \& Schmid, H. M. 1988, A\&A, 192, L10

Parimucha, Š., Arkhipova, V. P., Chochol, D., et al. 2000, Contrib. Astron. Obs. Skalnaté Pleso, 30, 99 [PAC]

Parimucha, Š. 2002, Ph.D. Thesis (in Slovak)

Schmid, H. M. 1998, Rev. Mod. Astron., 11, 297

Schild, H., \& Schmid, H. M. 1996, A\&A, 310, 211

Taranova, O. G., \& Yudin, B. F. 1983, A\&A, 117, 209

Taranova, O. G., \& Yudin, B. F. 1986, AZh, 63, 317

Taranova, O. G., \& Schenavrin, V. I. 2000, Astron. Lett., 26, 600

Theuns, T., \& Jorissen, A. 1993, MNRAS, 265, 946

Wallerstein, G. 1988, A\&A, 197, 161

Whitelock, P. A. 1987, PASP, 99, 573 